1 Estudiante de 5to año, Facultad de Medicina, Zaporozhye State Medical University.

2 Médico residente, Universidad de salud Dr. NTR, Vijayawada, India

3 Profesor Asociado, Departamento de Neurología, $6^{\circ} \mathrm{Hos}$ pital Municipal Municipal, Universidad Médica Estatal de Zaporozhye, Zaporozhye, Ucrania.

Palabras clave: Accidente Cerebrovascular, Tratamiento, Terapia trombolítica, Depresión, Calidad de vida.

Keywords: Stroke, thrombolytic therapy, Depression, Quality of Life.
Recibido para publicación: 12 de mayo 2018 Aceptado para publicación: 14 de julio 2018

Citar como:

Rev Cient Cienc Med 2018;21(1):30-39

\section{RESULTADOS DEL TRATAMIENTO DEL ICTUS ISQUÉMICO CON Y SIN ADMINISTRACIÓN DE TERAPIA TROMBOLÍTICA: UN ESTUDIO COMPARATIVO.}

\author{
Treatment outcomes of ischemic stroke with and without adminis- \\ tration of Thrombolytic therapy: a comparative study.
}

Varahabhatla Vamsi ${ }^{1}$, Vinisha Tekwani ${ }^{~}$, Vedula Ushakiranmayi ${ }^{2}$, Marina Sikorskaa ${ }^{3}$,

\section{RESUMEN}

Introducción: La terapia trombolítica intravenosa en la circulación cerebral, dentro de las primeras 3 horas del accidente cerebrovascular isquémico ofrece importantes beneficios netos para prácticamente todos los pacientes con déficit potencialmente incapacitantes.

Objetivo: Evaluar el curso de la enfermedad en pacientes con accidente cerebrovascular isquémico con una mejoría clínica del déficit neurológico durante el período de ventana de 3 horas en los grupos de pacientes que recibieron y no recibieron trombólisis intravenosa.

Métodos: Se realizó un estudio descriptivo, retrospectivo, donde revisamos los registros demográficos, clínicos, tomográficos, de procedimiento de los pacientes en el $6^{\circ}$ Hospital Clínico Municipal, Zaporozhye desde 2010 2017. Se analizaron un total de 78 historias clínicas, 12 eran pacientes con terapia trombolítica intravenosa y 66 con terapia tradicional. Los resultados del tratamiento se evaluaron sobre la base de la escala NIHSS y la escala de Rankin modificada después de 90 días después del tratamiento. Los datos se procesaron utilizando estadístico STATISTICA 7.0.

Resultados: En el grupo de pacientes con terapia trombolítica intravenosa, ninguno de los pacientes presentó un empeoramiento clínico. En el segundo grupo, 21 pacientes mostraron un aumento en el déficit neurológico en los primeros 3-5 días después de la hospitalización. La puntuación de NIHSS en el ler grupo, cuando se decidió sobre terapia trombolítica intravenosa y al alta durante 20 días, fue de $6 \pm 3,8$ y $0,5 \pm 0,2$, respectivamente. En el segundo grupo; $6 \pm 2,9$ y $4 \pm \mathrm{I}, \mathrm{I}$ I. De acuerdo con la escala de Rankine modificada, después de 3 meses en el primer grupo, el índice fue de $0,5 \pm 0, I$ ( $\leq I$ en 8 pacientes); en el segundo grupo $I \pm 0,3$.

Conclusiones: La trombólisis intravenosa en pacientes con regresión temprana espontánea de déficits neurológicos es aconsejable, y el fracaso no está justificado.

\section{ABSTRACT}

Introduction: Intravenous thrombolytic therapy at the cerebral circulation within the first 3 hours of ischemic stroke onset offers substantial net benefits for virtually all patients with potentially disabling deficits.

Aim: Evaluate the course of the disease in patients with ischemic stroke with a clinical improvement of the neurological deficit during the 3-hour window period in the groups of patients who received and did not receive intravenous thrombolysis.

Methods: A descriptive, retrospective study was performed, in which we reviewed demographical, clinical, tomographical, procedural records of the patients at 6th Municipal Clinical Hospital, Zaporozhye from 2010-2017.A total of 78 case histories were analyzed, 12 were patients with Intravenous thrombolytic therapy and 66 with traditional therapy. The results of the treatment were assessed on the basis of NIHSS scale and the Modified Rankin scale after 90 days after treatment. The statistical data was processed using STATISTICA ${ }^{\oplus}$ 7.0.

Results: In the group of patients with Intravenous thrombolytic therapy, none of the patients showed clinical worsening. In the 2nd group, 21 patients showed an increase in neurologic deficit in the first 3-5 days after hospitalization. The NIHSS score in the I stgroup, when deciding on Intravenous thrombolytic therapy and at discharge for 20 days, was $6 \pm 3,8$ and $0,5 \pm 0,2$, respectively. In the 2nd group; 6 $\pm 2,9$ and $4 \pm \mathrm{I}$, I I. According to the modified Rankine scale, after 3 months in the first group, the index was 0,5 $\pm 0, I$ ( $\leq I$ in 8 patients); in the second group I $\pm 0,3$.

Conclusions: Intravenous thrombolysis in patients with spontaneous early regression of neurological deficits is advisable, and failure is not justified. 


\section{MATERIALES Y MÉTODOS}

El ictus isquémico es una condición que se produce de- bido a la falta de suficiente suministro de sangre al cerebro. El accidente cerebrovascular isquémico en todo el mundo sigue siendo un importante problema médico y social debido a la alta incidencia de las tasas de discapacidad. El principal factor causal del accidente cerebral isquémico es la oclusión de la arteria cerebral, aunque puede variar en poblaciones ${ }^{2}$. Se estima que 15 millones de personas sufren de ictus cada año, de los cuales 1,2 millones de casos se registran en Europa, actualizada por la Organización Mundial de la salud. El Ministerio de Salud de Ucrania, en sus informes anuales, estima que alrededor de 100000 a 120000 personas admiten en la unidad de urgencias con un nuevo o recurrente accidente cerebrovascular cada año ${ }^{3}$. En un promedio el $85 \%$ se debe a infarto cerebral, $10 \%$ hemorragia primaria y un $5 \%$ hemorragia subaracnoidea. El tratamiento precoz es un factor importante en la terapia de reperfusión en el accidente cerebrovascular isquémico ${ }^{5}$. Se encontró muchos cambios sustanciales en el tratamiento de la apoplejía isquémica tras revelar la eficacia del tratamiento trombolítico, sobre la base de los resultados de muchas investigaciones ${ }^{6}$. Los métodos modernos de tratamiento y, en particular, el Tratamiento Trombolítico (TLT), están destinados a restablecer el flujo sanguíneo en los vasos afectados durante las primeras horas de la enfermedad, lo que contribuye a prevenir el desarrollo de un daño irreversible a la sustancia del cerebro o reducir su volumen y por lo tanto, minimizar el grado de déficit neurológico residual. La discapacidad posterior al accidente cerebrovascular ocupa el primer lugar entre sus causas y es de 3,2 por cada 10000 habitantes. Hasta un $20 \%$ de los pacientes regresan al trabajo, aproximadamente el $80 \%$ quedan discapacitados y el $25 \%$ de ellos necesitan ayuda externa ${ }^{7}$. La Trombólisis Intravenosa (IV) sistémica con un Activador de Plasminógeno Tisular recombinante ( $r$-tPA) es el método más eficaz para tratar el accidente cerebrovascular isquémico en las primeras 4,5 horas después del desarrollo de los síntomas. La Cooperativa Europea de estudios del accidente cerebrovascular agudo, "extendió el período de ventana de tiempo de 3 horas para el TLT de administración para el accidente cerebrovascular isquémico agudo a 4,5 horas" ${ }^{\prime \prime}$. Hacke W., et al. en su estudio aleatorizado, doble ciego, controlado con placebo de 620 pacientes, informó de los resultados positivos de la utilización de la trombolisis intravenosa con el activador del plasminógeno tisular recombinante para ictus hemisférico agudo, indicando las complicaciones de r-tPA en pacientes con la inelegibilidad ${ }^{9}$.

La alta eficiencia del método hace que sea razonable y necesaria para aumentar el número de pacientes en los cuales puede ser administrada. Una gran cantidad de contraindicaciones limitan significativamente el número de pacientes a los que se les administra con TLT.
Este estudio descriptivo, retrospectivo aleatorizado fue aprobado por el Comité de ética local de la Universidad Médica Estatal de Zaporozhye en el período comprendido entre 2010 y 2017. Se analizaron 78 historias clínicas de pacientes con ictus isquémico hemisférico agudo tratados en el departamento de trastornos de la circulación cerebral aguda del sexto hospital clínico de la ciudad de Zaporozhye. El tratamiento con trombolisis fue recibido por 12 pacientes (grupo 1), y la terapia estándar sin TLT - 66 pacientes (grupo 2). El criterio de inclusión fue una mejoría clínica de 1-2 puntos en la escala del accidente cerebrovascular del Instituto Nacional de Salud (NIHSS), en las primeras 3 horas después del inicio de la enfermedad. El activador tisular del plasminógeno (Actilyse ${ }^{\circledR}$ ) fue administrado a una velocidad de $0,9 \mathrm{mg} / \mathrm{kg}$ (no más de $90 \mathrm{mg}$ ), la solución del 10\% fue administrada para el bolo de 1 minuto, durante 60 minutos. Los resultados del tratamiento fueron evaluados en la escala NIHSS y en la escala modificada de Rankine en descarga y 90 días después del accidente cerebrovascular. El accidente cerebrovascular isquémico fue verificado por Tomografia Computarizada (TC), repetida en 7 pacientes en el primer grupo y en 43 pacientes en el segundo grupo. En el primer grupo, todos los pacientes que ingresaron a la clínica dentro de 2-3 horas después de la aparición de la enfermedad. Los indicadores demográficos y tomográficas fueron evaluados. Los datos estadísticos se procesaron utilizando STATISTICA ${ }^{\circledR}$ 7.0, con los programas estándar.

\section{RESULTADOS}

En el primer grupo, todos los pacientes ingresaron a la clínica dentro de las 2-3 horas desde el inicio de la enfermedad. En la escala de NIHSS los pacientes tenían de 3 a 8 puntos (un promedio de 5,85), como se muestra en la Fig. 1. Todos los pacientes en el estado neurológico tenían paresias de diversos grados de severidad y 4 pacientes tenían afasia motora. Todos los pacientes notaron alguna mejoría en la condición hasta que ingresaron a la clínica con un aumento en el volumen de movimientos activos en una o dos extremidades (un promedio de 1-2 puntos en la escala NIHSS), un paciente notó una mejoría en el habla. Debido al hecho de que la mejoría de la condición no fue rápida, y en ningún caso hubo una regresión completa de los síntomas neurológicos, se tomó la decisión de realizar TLT. De acuerdo con la TC, no se observaron signos de la formación del foco. El tiempo desde el inicio de la enfermedad hasta el ingreso a la clínica fue de 105 minutos en promedio. El tiempo desde el ingreso a la clínica antes del inicio del tratamiento es de 50 a 70 minutos.

Al momento del alta, el puntaje promedio en la escala NIHSS en este grupo fue de 0,93 puntos, el nivel de independencia funcional se evaluó de acuerdo con la escala de Rankine modificada en la Fig. 2, finalmente, en este grupo de pacientes 
obtuvo 0,5 puntos (mientras que 7 pacientes obtuvieron 0 puntos).

En el momento de la admisión, el puntaje total en el segundo grupo fue de 6,32, el curso de la enfermedad estuvo marcado por un polimorfismo significativo: en 9 pacientes $(13,6 \%)$ el déficit neurológico retrocedió por completo durante 2-6 días, en $36(54,5 \%)$ pacientes no mostraron un déficit neurológico que empeorara, dinámica positiva, y al extracto en la escala NIHSS no excedió 2,5 puntos, y el nivel de independencia funcional promedió 1,3 puntos en la escala de Rankine. Además, 21 pacientes $(31,8 \%)$ tuvieron un aumento en los síntomas neurológicos en promedio de

Figura 1: Dinámica de la severidad de la enfermedad cerebrovascular isquémica hemisférica cerebral en pacientes en los primeros 3 meses de la enfermedad (según la escala NIHSS).

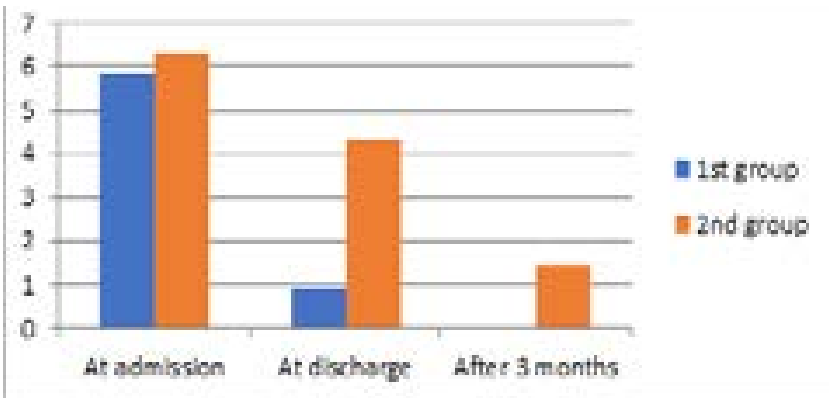

Figura 2: Dinámica de la discapacidad y la independencia funcional de los pacientes con isquemia hemisférica cerebral en los primeros 3 meses de la enfermedad (escala Rankine modificado) dependiendo del tipo de terapia.

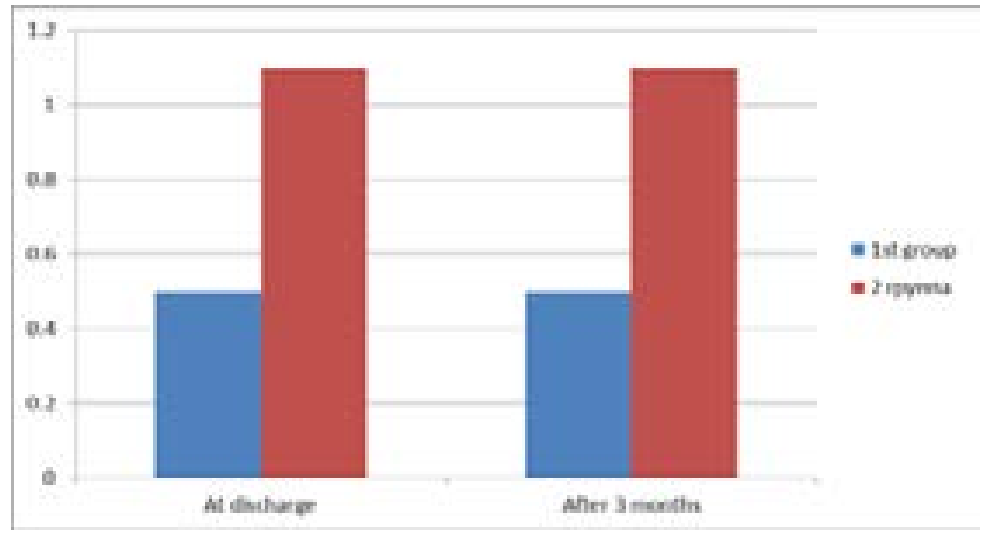

Figura 3: Dinámica de los trastornos depresivos en pacientes con enfermedad cerebrovascular isquémica hemisférica cerebral en los primeros 3 meses de la enfermedad (DASS escala).

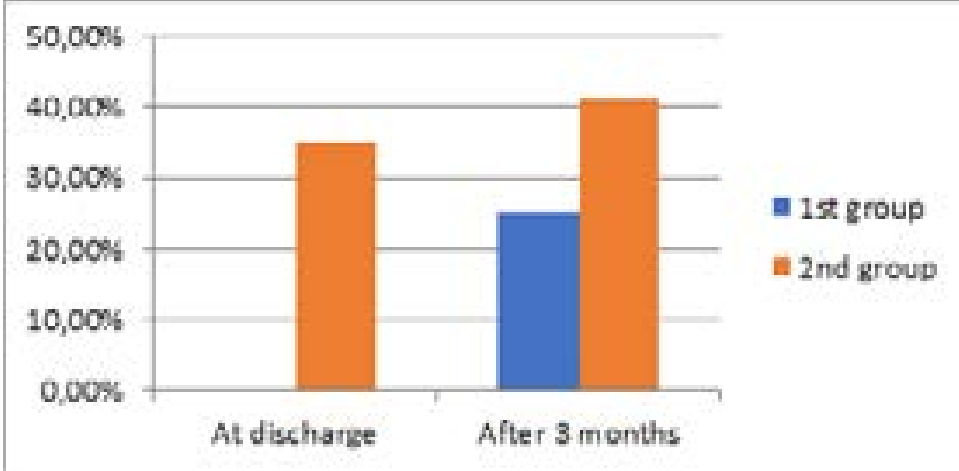

32
4,1 puntos en la escala NIHSS en el transcurso de 5 días en comparación con el estado de admisión. Posteriormente, se observó la regresión de los síntomas neurológicos y para el alta el NIHSS de estos pacientes tenían un promedio de 8,1 puntos, y de acuerdo con la escala de Rankine modificada, 1,8 puntos.

El puntaje total en el segundo grupo de pacientes en la escala NIHSS fue de 4,3 y en el puntaje de Rankine 1,1. La evaluación de los resultados en el tratamiento en el día 90 desde el inicio de la enfermedad fue una complicación significativa, ya que los pacientes generalmente estaban en casa. 8 pacientes del primer grupo y 22 del segundo grupo fueron examinados en la clínica, la información de los pacientes restantes o sus familiares se obtuvo como resultado de una entrevista telefónica, lo que reduce la fiabilidad de los resultados obtenidos. En el primer grupo de pacientes, no hubo resultados letales de la enfermedad. En la escala NIHSS el puntaje promedio fue 0 (los pacientes tenían 0-1 puntos), en la escala de Rankin el puntaje fue de 0,5; con 8 pacientes menos de 1 punto.

En el segundo grupo, se observó la muerte de un paciente (accidente cerebrovascular isquémico repetido 2,5 meses después de que se transfirió el primero). El puntaje total de NIHSS disminuyó a 1,75 puntos debido a la recuperación de trastornos motores y afásicos, y en la escala de Rankin 1,0 punto. Además, se analizaron los trastornos depresivos que ocurrieron en los pacientes dentro de los 3 meses posteriores a un accidente cerebrovascular. La depresión post-ictus ralentizará significativamente el proceso de recuperación, empeorando la calidad de la rehabilitación de los pacientes. La presencia de depresión se determina usando la Escala de Depresión Ansiedad Estrés (DASS), como se muestra en la Fig. 3. En el momento del alta, los pacientes en el primer grupo mostraron una ausencia de depresión. En el segundo grupo, 43 pacientes $(65,2 \%)$ no tenían depresión (puntuaciones 0-9 DASS), un grado leve (10-13) de trastornos en 13 pacientes $(19,7 \%)$ y trastornos depresivos moderados (14-20 puntos) en 10 pacientes $(15,1 \%)$.

Después de 3 meses, se observaron signos de depresión leve en DASS en 3 pacientes del grupo 1 (25\%). En el segundo grupo, hubo una disminución en el número de pacientes sin trastornos depresivos a 39 personas (59\%), un aumento en los pacientes con trastornos depresivos leves de 13 a 18 pacientes $(27,3 \%)$ y 9 pacientes mostraron un grado moderado de depresión (13,7\%). También se observó que los trastornos depresivos se observaron predominantemente en personas con sintomatología neurológica focal que al parecer reflejaba la calidad de vida de los pacientes como consecuencia, el aumento de los trastornos depresivos. 


\section{DISCUSIÓN}

En este estudio, en el primer y el segundo grupo, no hubo dinámica desde el momento del alta y 3 meses después del desarrollo de la enfermedad. Las causas en los grupos están algo marcadas. Creyendo que el proceso de restauración de funciones continuaría por sí mismo.

Los pacientes del primer grupo se recuperaron fácilmente de las alteraciones motoras y afásicas durante su estancia en el hospital, prácticamente no participaron en una sesión de terapeuta del habla y rehabilitadora. En el segundo grupo, los pacientes se sometieron a un curso de rehabilitación, ambos en el hospital (Departamento de Neurorrehabilitación) y ambulatorio (en clínicas de rehabilitación o individualmente con especialistas en rehabilitación), lo cual se confirma con los índices NIHSS, sin embargo, la mayoría de los pacientes antes de la jubilación y jubilados notaron que la ayuda de familiares era necesaria, porque tienen miedo de salir de casa por sí mismos, preparar la comida, lavar la ropa y bañase. En nuestra opinión, se debe principalmente a problemas psicológicos. En primer lugar, garantiza visitas frecuentes a niños y nietos, una mayor atención de los familiares $y$, en segundo lugar, facilita la calidad de vida en estos pacientes.

La dinámica de los trastornos depresivos es muy interesante. En el primer grupo de pacientes, al cabo de 3 meses, el número de violaciones alcanzó el $25 \%$ debido a la aparición de muchos trastornos al alta. Se puede suponer que encontrar una propiedad (no todos los pacientes trabajaban), el aislamiento en el pasatiempo, la disminución de la comunicación social con amigos contribuyó a la aparición del síndrome depresivo. En el segundo grupo hay un aumento en el número total de pacientes con depresión del $34,8 \%$ al $41 \%$, con una disminución en el número de pacientes sin signos de depresión y con gravedad moderada, y el número de pacientes con trastornos depresivos leves (de 19,8\% a 27,3\%). Estas causas pueden considerarse características del primer grupo, pero la presencia de un déficit motor y un mayor grado de dependencia funcional son adicionales.

Marten G., et al. en su estudio mencionó que, en pacientes con accidente cerebrovascular isquémico agudo se puede iniciar el tratamiento dentro de las 3 horas o 4,5 horas desde el inicio de los síntomas por vía IV (r-tPA) de un sobre sin usar ${ }^{10}$.

Kim JT., et al. declararon que la trombólisis iniciada dentro de los primeros 60 minutos (hora de oro) de ataque se asocia con resultados positivos en pacientes con accidente cerebrovascular isquémico agudo ${ }^{11}$.

Emberson J., et al. con 6756 pacientes y 9 ensayos clínicos aleatorizados mencionaron que alteplasa es eficaz en el tratamiento del accidente cerebrovascular isquémico agudo, especialmente en pacientes de mayor edad, así como en pacientes con accidentes cerebrovasculares recurrentes.
El autor postuló los efectos beneficiosos del uso de alteplasa con 4,5 horas de inicio del accidente cerebrovascular. También compararon los resultados con y sin la administración de alteplasa y establecieron el resultado positivo de su administración ${ }^{12}$.

Logallo N., et al. con 1100 pacientes realizaron un ensayo aleatorizado ciego que indicó que tenecteplasa es un nuevo agente trombolítico con algunos beneficios farmacológicos en comparación con alteplasa, pero son necesarios más ensayos clínicos para demostrar los beneficios y el uso de tenecteplasa sobre alteplasa ${ }^{13}$.

Breuer, et al. en su estudio señalo que no había beneficios específicos después de la terapia trombolítica en pacientes que se sometieron a cribado en Imágen de Resonancia Magnética (IRM) y en pacientes sin cribado en IRM ${ }^{14}$.

Tsivgoulis, et al. en su metanálisis mostraron que el uso de ultrasonido de alta frecuencia puede lograr una recanalización completa de las arterias en comparación con solo rt-PA ${ }^{15}$.

Sandercock, et al. en su estudio sobre los participantes 3035 , declaró los resultados de la tercera carrera internacional trial (IST-3), administradas vía IV alteplasa dentro de las 6 horas del inicio del movimiento, mejorar el funcionamiento en los pacientes ${ }^{16}$.

Anderson, et al, realizaron el estudio Encantada, para comparar la dosis de alteplasa con dosis estándar, con la dosis mas baja de su administración. Trabajaron con la hipótesis que menores dosis de alteplasa, pueden posiblemente reducir el riesgo de hemorragia intracerebral y mejorar la recuperación ${ }^{17}$.

Rothwell P, en su revisión en la ronda del 2016 mencionó diferentes estudios sobre el accidente cerebrovascular y su gestión en un entorno clínico. Él discutió sobre el ensayo ATACH-2 y el ensayo INTERACT-2 centrada en reducir moderadamente la presión sanguínea sistólica, lo que aumenta la seguridad, reducir la discapacidad en los pacientes con ictus agudo. Una alta presión sistólica de $180 \mathrm{~mm} / \mathrm{Hg}$ se redujo nicardipino vía IV, a un promedio de $140 \mathrm{~mm} / \mathrm{Hg}^{18}$.

Sandercock P y Ricci en su revisión sistemática confirman claramente que el tiempo es el cerebro como la prioridad para el reconocimiento de estos pacientes y el transporte rápido al hospital durante el periodo de ventana de oro, seguida por la clínica y radiológia y pronta del TLT. Estos podrían reducir los déficits neurológicos y aumentar la calidad de vida².

Moussaddya, et al. en su revisión sistemática, debatieron las consideraciones futuras sobre la terapia del accidente cerebrovascular. Describieron la angiografía y trombectomía mecánica mediante stents retrievers que especifican la composición del trombo, jugando un papel potencial en el diagnóstico clínico e inicio de protocolos de tratamiento urgente. La introducción de tratamientos pre-ambulatorios se han destacado en su literatura, los cuales podrían servir como unidades móviles modernos de ictus isquémico equi- 
pado con un escáner de TC y agentes trombolíticos ${ }^{19}$.

Es interesante que los pacientes del primer grupo notaran el hecho de llevar a cabo el método más moderno y costoso de tratamiento del ictus isquémico, lo que les inspiró confianza en el resultado positivo de la terapia y la recuperación. Después de un análisis comparativo de los resultados del tratamiento de los pacientes, se puede observar que la gravedad del déficit neurológico en la escala NIHSS es más pronunciada y rápida en los pacientes del grupo 1 (Fig. 1), sin embargo, en el segundo grupo hay una dinámica positiva persistente (de 6,32 a 1,45 puntos), que puede explicarse por el hecho de que todos los pacientes tuvieron una enfermedad leve en el debut y la efectividad de las medidas de rehabilitación.

\section{Conclusiones:}

Tanto en el primer grupo como en el segundo, las personas mayores eran más susceptibles a la depresión. Los resultados sugieren que la dinámica del grado de déficit neurológico y la independencia funcional de los pacientes es más pronunciada en el grupo de pacientes donde el tratamiento

\section{REFERENCIAS}

1.Bivard A, Levi C, Krishnamurthy V, et al. Perfusion computed tomography to assist decision making for stroke thrombolysis. Brain. 2015 July 1;138(7): 1919-31.

2.Sandercock P, Ricci S. Controversies in Trombolysis. Curr Neurol Neurosci Rep. 2017 Jun 30;17(8):60. Doi: 10.1007/ s11910-017-0767-5. PubMed PMID: 28667504; PubMed Central PMCID: PMC5493717

3.O.O. Filipets, V.M. Pashkovsky. Stroke burden in ukraine: analysis of the official stroke statistics and overview of population-based epidemiological studies. Clin. and experim. Pathol. 2014;13(3 Suppl 49):189-93.

4.Royal college of physicians, National clinical Guideline for stroke prepared by intercollegiate stroke working party. $2016 ; 5^{\text {th }}$ Edition. Available from: https://www.strokeaudit.org/SupportFiles/ Documents/Guidelines/2016-National-Clinical-Guideline-forStroke-5t-\%281\%29.aspx

5.Stroke, Available from: http://www.strokecenter.org/patients/ about-stroke/what-is-a-stroke/.

6.Luciana C, Joseph T, Marc F. Acute Ischemic Stroke Therapy Overview. In stroke compendium of Clinical Research page 541-558. February 3, 2017.

7.Bernhard N, Christoph K, Guido S et al. Ischaemic stroke: a thrombo-inflammatory disease. J Physiol. 2011 Sep 1; 589(Pt 17): 4115-23.

8.Milija D. Mijajlovic. Thrombolytic or endovascular therapy for acute ischemic stroke: Time is brain. J Neurosci Rural Pract. 2014 Jan-Mar; 5(1): 3-5.

9.Hacke W, Kaste M, Fieschi C et.al. Intravenous thrombolysis with recombinant tissue plasminogen activator for acute hemispheric stroke. The European Cooperative Acute Stroke Study (ECASS), JAMA. 1995 Oct 4; 274(13):1017-25.

10.Maarten G, Lansberg, Martin J, et al. Antithrombotic and Thrombolytic Therapy for Ischemic Stroke, Antithrombotic Therapy and Prevention of Thrombosis, 9th ed: American College of Chest Physicians Evidence-Based Clinical Practice Guidelines, Chest. 2012 Feb; 141(2 Suppl): e601S-e36S. del ictus hemisférico isquémico se realizó con el uso de terapia trombolítica $y$, en consecuencia, la trombólisis intravenosa en pacientes con la regresión temprana espontánea del déficit neurológico es conveniente, y el fracaso no siempre está justificado.
11.Kim JT; Fonarow GC; Smith EE, et al. Treatment With Tissue Plasminogen Activator in the Golden Hour and the Shape of the 4.5-Hour Time-Benefit Curve in the National United States Get With The Guidelines-Stroke Population. 2017; 135(2):128-139. 12.Emberson J; Lees KR; Lyden P, et al. Effect of treatment delay, age, and stroke severity on the effects of intravenous thrombolysis with alteplase for acute ischaemic stroke: a meta-analysis of individual patient data from randomized trials. Lancet. 2014; 384(9958):1929-35.

13.Logallo N; Novotny V; Assmus J, et al. Tenecteplase versus alteplase for management of acute ischaemic stroke (NORTEST): a phase 3, randomized, open-label, blinded endpoint trial. Lancet Neurol. 2017; 16(10):781-8.

14.Breuer L., Schellinger P.D., Huttner H.B., et al. Feasibility and safety of magnetic resonance imaging-based thrombolysis in patients with stroke on awakening: Initial single-centre experience. Int J Stroke. 2010;5: 68-73

15.Tsivgoulis G., Eggers J., Ribo M., et al. Safety and efficacy of ultrasound-enhanced thrombolysis: A comprehensive review and meta-analysis of randomized and nonrandomized studies. Stroke. 2010;41: 280-287.

16.Sandercock P, Wardlaw JM, Lindley RI, Dennis M, Cohen G, Murray $\mathrm{G}$, et al. The benefits and harms of intravenous thrombolysis with recombinant tissue plasminogen activator within $6 \mathrm{~h}$ of acute ischaemic stroke (the third international stroke trial [IST-3]): a randomized controlled trial. Lancet. 2012;379(9834):2352-2363.

17.Anderson CS, Robinson T, Lindley RI et al. Low-dose versus standard-dose intravenous alteplase in acute ischemic stroke. NEJM. 2016; 374(24):2313-2323.

18. Rothwell PM. Stroke research in 2016: when more medicine is better, and when it isn't. LancetNeurol. 2017;16(1):2-3.

19.Moussaddya A, Demchuk A.M, Hill M.D. Invited review. Thrombolytic therapies for ischemic stroke: Triumphs and future challenges. Neuropharmacology.2018 May.134 (Part B):272-9. 